NASA/TM-2004-212970

\title{
Generalization of Equivalent Crystal Theory to Include Angular Dependence
}

John Ferrante

Glenn Research Center, Cleveland, Ohio

Fredy R. Zypman

Yeshiva University, New York, New York 
Since its founding, NASA has been dedicated to the advancement of aeronautics and space science. The NASA Scientific and Technical Information (STI) Program Office plays a key part in helping NASA maintain this important role.

The NASA STI Program Office is operated by Langley Research Center, the Lead Center for NASA's scientific and technical information. The NASA STI Program Office provides access to the NASA STI Database, the largest collection of aeronautical and space science STI in the world. The Program Office is also NASA's institutional mechanism for disseminating the results of its research and development activities. These results are published by NASA in the NASA STI Report Series, which includes the following report types:

- $\quad$ TECHNICAL PUBLICATION. Reports of completed research or a major significant phase of research that present the results of NASA programs and include extensive data or theoretical analysis. Includes compilations of significant scientific and technical data and information deemed to be of continuing reference value. NASA's counterpart of peerreviewed formal professional papers but has less stringent limitations on manuscript length and extent of graphic presentations.

- TECHNICAL MEMORANDUM. Scientific and technical findings that are preliminary or of specialized interest, e.g., quick release reports, working papers, and bibliographies that contain minimal annotation. Does not contain extensive analysis.

- CONTRACTOR REPORT. Scientific and technical findings by NASA-sponsored contractors and grantees.
- CONFERENCE PUBLICATION. Collected papers from scientific and technical conferences, symposia, seminars, or other meetings sponsored or cosponsored by NASA.

- SPECIAL PUBLICATION. Scientific, technical, or historical information from NASA programs, projects, and missions, often concerned with subjects having substantial public interest.

- TECHNICAL TRANSLATION. Englishlanguage translations of foreign scientific and technical material pertinent to NASA's mission.

Specialized services that complement the STI Program Office's diverse offerings include creating custom thesauri, building customized databases, organizing and publishing research results ... even providing videos.

For more information about the NASA STI Program Office, see the following:

- Access the NASA STI Program Home Page at http://www.sti.nasa.gov

- E-mail your question via the Internet to help@sti.nasa.gov

- Fax your question to the NASA Access Help Desk at 301-621-0134

- Telephone the NASA Access Help Desk at 301-621-0390

- Write to:

NASA Access Help Desk

NASA Center for AeroSpace Information 7121 Standard Drive

Hanover, MD 21076 
NASA/TM-2004-212970

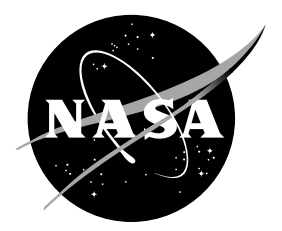

\section{Generalization of Equivalent Crystal Theory to Include Angular Dependence}

John Ferrante

Glenn Research Center, Cleveland, Ohio

Fredy R. Zypman

Yeshiva University, New York, New York

National Aeronautics and

Space Administration

Glenn Research Center 


\section{Acknowledgments}

We would like to thank the Tribology and Surface Science Branch, NASA Glenn Research Center, where part of the work was performed. We also thank Dr. Phillip Abel for fruitful comments.

This work was sponsored by the Low Emissions Alternative

Power Project of the Vehicle Systems Program at the

NASA Glenn Research Center.

Available from

NASA Center for Aerospace Information 7121 Standard Drive

Hanover, MD 21076
National Technical Information Service 5285 Port Royal Road

Springfield, VA 22100

Available electronically at http:/ /gltrs.grc.nasa.gov 


\title{
Generalization of Equivalent Crystal Theory to Include Angular Dependence
}

\author{
John Ferrante \\ National Aeronautics and Space Administration \\ Glenn Research Center \\ Cleveland, Ohio 44135 \\ Fredy R. Zypman \\ Yeshiva University \\ Department of Physics \\ New York, New York 10033-3200
}

\begin{abstract}
In the original Equivalent Crystal Theory, each atomic site in the real crystal is assigned an equivalent lattice constant, in general different from the ground state one. This parameter corresponds to a local compression or expansion of the lattice. The basic method considers these volumetric transformations and, in addition, introduces the possibility that the reference lattice is anisotropically distorted. These distortions however, were introduced ad-hoc. In this work, we generalize the original Equivalent Crystal Theory by systematically introducing site-dependent directional distortions of the lattice, whose corresponding distortions account for the dependence of the energy on anisotropic local density variations. This is done in the spirit of the original framework, but including a gradient term in the density. This approach is introduced to correct a deficiency in the original Equivalent Crystal Theory and other semiempirical methods in quantitatively obtaining the correct ratios of the surface energies of low index planes of cubic metals-(100), (110), (111). We develop here the basic framework, and apply it to the calculation of $\mathrm{Fe}(110)$ and $\mathrm{Fe}$ (111) surface energy formation. The results, compared with first principles calculations, show an improvement over previous semiempirical approaches.
\end{abstract}

\section{Introduction}

Equivalent Crystal Theory (ECT) is a method to calculate energies of atomic conglomerates. It has been very successful in predicting formation energies of bulk defects. Bozzolo, Ferrante and Smith (BFS) have extended it to multicomponent metals, and have successfully used it to predict the ground state structures and surface properties of various alloys.

The basic idea of the original Equivalent Crystal Theory (OECT) rests on the fact that the total ground state energy of a quantum system is a functional of the electron density [1]. Therefore, OECT requires that, at the atomic site under consideration, the density be the same for both the real lattice and the equivalent, defect-free lattice. That provides a value for the equivalent lattice parameter $\mathrm{a}_{\mathrm{eq}}$ at that site. The energy contribution from that site is obtained by using the Universal Binding Energy Relation (UBER) curve. The UBER provides binding energy as a 
function of average atomic separation (or in this case, lattice parameter). In this work, we generalize OECT without losing its original flavor, that is, by keeping the assumption that the electron density must remain the same for both the real and the equivalent crystal. However, here we extend it to include non-local effects by requiring that both, the real and equivalent crystal densities be the same not only at the center of the atomic site into consideration, but also in a neighborhood around that point. Concretely, we will impose that the density at an atomic site and its gradient be the same for the real and the equivalent crystal. These density variations in the neighborhood can be estimated from recent ab initio calculation for surface binding curves for Fe. The reference crystal now includes both isotropic distortions and separation of the solid in two pieces normal to a given surface plane.

In this new framework, the reference crystal at an atomic site will be assigned two (instead of one) parameters: the equivalent lattice parameter and the relative separation, $\gamma$, between two hypothetical surfaces. The direction of the separation, and the separation itself, do not have to be the same for all the real atoms in the crystal. We will call that, potentially variable, direction $\mathrm{z}$. The two parameters are obtained from two requirements, which are: the site density and its gradient at each location are assumed the same for both the real and the equivalent crystal.

\section{Development of the Ideas}

In this modified version of ECT (MECT), the reference crystal is not just a perfect lattice with $\mathrm{a} \neq \mathrm{a}_{0}$, but, in addition, it incorporates a pair of surface-terminated bulks with $\mathrm{a} \neq \mathrm{a}_{0}$, separated by a distance $\gamma d$, where $d$ is the interplanar distance normal to a particular plane.

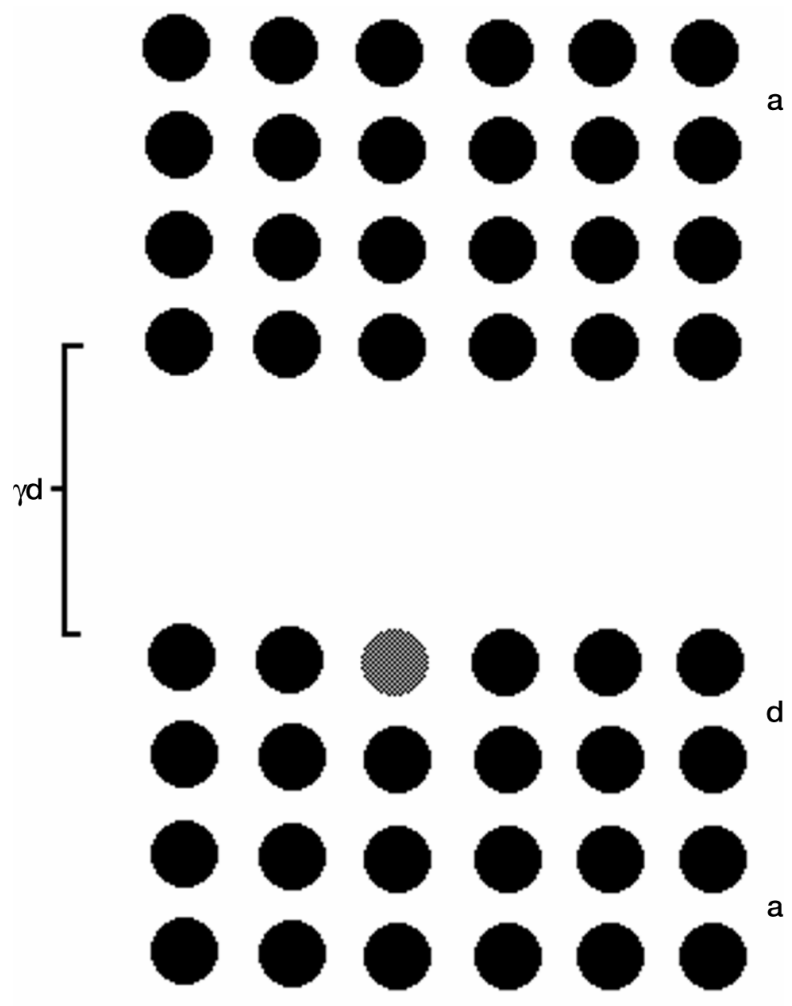

Figure 1.-Reference crystal. 
Figure 1 shows the reference crystal in a generic configuration. Clearly, as opposed to the original ECT (OECT), not all atoms in this reference crystal contribute the same amount to the total energy. We will consider the local energy contribution coming from a reference atom in the (quasi) surface (QS), shown in red in the figure. The quasi-surface becomes a bona fide surface in the limit $\gamma \rightarrow+\infty$. An atom in QS (AQS) contribution to the total energy is now a function of both the lattice parameter a, and the relative distance $\gamma$, between the two QS. This differs in form from OECT which expands the energy in a series containing a volume term, plus two-, three-, and four-body terms. In this formulation, the next term beyond the volume's would be the gradient of the density, and the next beyond that would be the curvature.

In OECT, a was found by requiring that the total electron density at the center of a given atom be the same for both the real defect crystal and the reference crystal. That condition will remain valid in MECT, but we will impose a second constraint. The motivation for the additional equation is to also look at the density close to the center of the given atom, not only at the center. We will impose that the real and reference densities must be equal in that small (but not zero) region. As in OECT, we take the densities from each atom to be given by $\rho(\vec{r})=\sum_{\vec{R}}|\vec{R}-\vec{r}|^{p} e^{-\alpha|\vec{R}-\vec{r}|}$, with standard notation. Consider the atom for which we want to calculate the energy contribution, to be located at the origin. We expand $|\vec{R}-\vec{r}|$ taking into account that $\mathrm{r}<<\mathrm{R}$ :

$$
\rho(\vec{r})=\rho(\overrightarrow{0})+\left.\vec{r} \cdot \nabla \rho(\vec{r})\right|_{\vec{r}=\overrightarrow{0}}+\cdots
$$

Then, expanding the power and the exponential,

$$
\rho(\vec{r}) \approx \sum_{\vec{R}} R^{p} e^{-\alpha R}+\vec{r} \cdot \sum_{\vec{R}}(\alpha R-p) R^{p-1} e^{-\alpha R} \frac{\vec{R}}{R}
$$

From this expression, we will impose:

$$
\begin{gathered}
\sum_{\vec{R} \text { equivalent }} R^{p} e^{-\alpha R}=\sum_{\vec{R} \text { real }} R^{p} e^{-\alpha R} \\
{\left[\sum_{\vec{R} \text { equivalent }}(\alpha R-p) R^{p-1} e^{-\alpha R} \frac{\vec{R}}{R}\right] \cdot \hat{n}_{e}=\left[\sum_{\vec{R} \text { real }}(\alpha R-p) R^{p-1} e^{-\alpha R} \frac{\vec{R}}{R}\right] \cdot \hat{n}_{r}}
\end{gathered}
$$

where $\hat{n}_{e}$ and $\hat{n}_{r}$ are fixed directions in the respective spaces.

Both right-hand sides are explicit functions of $\gamma$ and $\underline{a}$ and will be obtained by solving (3) and (4). 


\section{Generalization of UBER}

The most critical issue to stress in MECT is the fact that, as opposed to what happens in OECT, not all atoms in the reference crystal are equivalent and therefore contribute a different amount to the total energy.

Let us assign the energy contribution from each atom to the total energy

$$
E_{n}=E_{A Q S}+\left(E_{B}-E_{A Q S}\right)\left[1-e^{-\lambda(n-1)}\right]
$$

where all atoms in the same plane $\mathrm{n}$ will be weighted equally, but atoms in different layers will have a different contribution. Here $\mathrm{E}_{\mathrm{B}}$ is the contribution from bulk (which is given by the OECT UBER), $\mathrm{E}_{\mathrm{AQS}}$ is the (still unknown) contribution from $\mathrm{AQS}, \lambda \approx 1 / \mathrm{M}(\mathrm{M}$ is the number of layers in which atoms are substantially different from bulk), finally $\mathrm{n}$ is the layer's depth ( $\mathrm{n}=1$ corresponds to the top layer, our QS). Here we have assumed an exponential form for the contribution to the energy as a function of depth. Hung et al [2] have shown that the electron density in slabs of increasing widths rapidly approaches its bulk value .

The total energy $\mathrm{E}_{\text {tot }}$ can be calculated as:

$$
E_{\text {Tot }}=N_{A} \sum_{n=1}^{+\infty} E_{n}=N_{A} \cdot N_{D} \cdot E_{B}+N_{A} \cdot\left(E_{A Q S}-E_{B}\right) \cdot \sum_{n=1}^{+\infty} e^{-\lambda(n-1)}
$$

Where $\mathrm{N}_{\mathrm{A}}$ is the number of atoms in-plane, $\mathrm{N}_{\mathrm{D}}$ is the number of layers (although the sum goes to infinity here, in practice $\mathrm{N}_{D}$ is very large but not infinite).

Calling $\mathrm{N}=\mathrm{N}_{\mathrm{A}} \cdot \mathrm{N}_{\mathrm{D}}$, the total number of atoms, we obtain:

$$
E_{T o t}=N \cdot E_{B}+\frac{N_{A} \cdot\left(E_{A Q S}-E_{B}\right)}{1-e^{-\lambda}}
$$

Hung et al [3] have done a first-principles density functional theory calculation where they obtained the energy as a function of separation of two surface-terminated bulks. Call that energy curve $\mathrm{E}\left(\gamma \mathrm{d}_{0}\right)$, where $\mathrm{d}_{0}=\mathrm{ca}_{0}$ is the equilibrium interplanar distance, $\mathrm{a}_{0}$ is the equilibrium lattice parameter, and $\mathrm{c}$ is a geometric factor that depends on the orientation and the crystal structure. Ferrante and Smith [4] defined the adhesive energy as the energy two surfaces have referred to the energy they have when infinitely separated. Since reference [1] reports energy per unit area (A), we have, for one QS:

$$
E_{a d}(\gamma) \equiv \frac{E(\gamma)-E(+\infty)}{A}
$$

We now make the identification $\mathrm{E}_{\mathrm{Tot}}=2 \mathrm{E}$ in equations (7) and (8), since they both correspond to total energies of the two QSs, calculated independently. Then, 


$$
E_{a d}(\gamma)=\frac{2 N_{A}}{A}\left[\frac{E_{A Q S}(\gamma)-E_{B}}{1-e^{-\lambda}}-\frac{E_{A Q S}(+\infty)-E_{B}}{1-e^{-\lambda}}\right]
$$

When $\gamma=1$, the two QSs are separated by their equilibrium distance, and then $\lambda=0$ and $\mathrm{E}_{\mathrm{AQS}}=\mathrm{E}_{\mathrm{B}}$. Thus, in that case:

$$
E_{a d}(1)=\frac{2 N_{A}}{A} \frac{E_{B}-E_{A Q S}(+\infty)}{1-e^{-\lambda}}
$$

and

$$
E_{A Q S}(+\infty)=E_{B}+\frac{A}{2 N_{A}}\left(e^{-\lambda}-1\right) E_{a d}(1)
$$

Substituting (11) into (9):

$$
E_{a d}(\gamma)=\frac{2 N_{A}}{A} \frac{E_{A Q S}(\gamma)-E_{B}}{1-e^{-\lambda}}+E_{a d}(1)
$$

Solving for $\mathrm{E}_{\mathrm{AQS}}(\gamma)$, which is the energy of the reference atom AQS,

$$
E_{A Q S}(\gamma)=E_{B}+\frac{A}{2 N_{A}}\left(1-e^{-\lambda}\right)\left[E_{a d}(\gamma)-E_{a d}(1)\right]
$$

Call $\Gamma$ the area occupied by an AQS, that is $\Gamma \equiv \frac{A}{N_{A}}$. If $\mathrm{E}_{\mathrm{B}}$ is the energy per atom, then the energy per 2D Wigner-Seitz area is:

$$
E_{A Q S}(\gamma)=\frac{E_{B}}{\Gamma}+\frac{1}{2}\left(1-e^{-\lambda}\right)\left[E_{a d}(\gamma)-E_{a d}(1)\right]
$$

The term $\mathrm{E}_{\mathrm{B}}$ has been parameterized [5] by the Universal Binding Energy Relation (UBER):

$$
E_{B}(x)=E_{c}\left[1-\left(1+a^{*} e^{-a^{*}}\right)\right]
$$

where $x=\frac{a}{a_{0}}$, is the relative lattice parameter of the reference crystal, $\mathrm{E}_{\mathrm{c}}$ is the bulk cohesive energy, and:

$$
a^{*}=\frac{a}{\ell_{B}}\left(\frac{3}{8 \pi}\right)^{\frac{1}{3}}(x-1)
$$

for BCC structures. Here $\ell_{\mathrm{B}}$ is related with the bulk modulus and is known for metals [6,7], and a is the lattice parameter.

$E_{a d}(\gamma)$ in equation (12) has also been parameterized by a UBER in reference [1]: 


$$
E_{a d}(\gamma)=-2 \gamma_{S}\left[1+\frac{d}{\ell_{S}}(\gamma-1)\right] e^{-\frac{d}{\ell_{S}}(\gamma-1)}
$$

It is important to note that the last expression is valid only for $\mathrm{a}=\mathrm{a}_{0}$ since that is the calculation performed in reference [1]. We need to extend it to any value of $\gamma$ and a.

If they had done the same (adhesion) calculation for a crystal with a larger lattice parameter, the energy (for a given relative separation) would have been smaller. In particular, in the limiting case $\mathrm{a} \rightarrow+\infty$, the energy of the system tends to zero, for any separation. On the other hand, the first principles calculation $\mathrm{A}(\gamma)$ (explicitly written in terms of the relative separation) has a minimum for $\gamma=1$, that is for a separation equal to the lattice parameter (or the corresponding interplane distance). Finally, we write the excess (referred to the bulk ground state) energy of the reference atom AQS as:

$$
\Delta E_{A Q S}=E_{c}\left[1-\left(1+a^{*} e^{-a^{*}}\right)\right]+\gamma_{S}\left\{1-\left[1+\frac{x d_{0}}{\ell_{S}}(\gamma-1)\right] e^{-\frac{x d_{0}}{\ell_{S}}(\gamma-1)}\right\} e^{-a^{*}}
$$

This expression is built in such a way that the ground state of the system corresponds to $\gamma=1$ and $\mathrm{x}=1$, that is an undistorted crystal with the equilibrium lattice parameter. Any configurational change, away from that structure, can only increase the energy.

\section{Setting the Reference QS (100)}

In this section, we build a reference crystal as a BCC (100) QS.

Figure 2 shows the geometry for the BCC (100) QS. We take a "unit cell" in which the atoms below the QS are allowed to move under isotropic transformations. The atoms above the QS, can support both volume and surface-surface separation transformation. Nearest neighbors to AQS are denoted $\vec{R}$ and $\vec{R}_{\gamma}$. A typical $\vec{R}_{\gamma}$ (out of four) is $\vec{R}_{\gamma}=a\left(1,1, \frac{1+\gamma}{2}\right)-\frac{1}{2} a(1,1,1)=\frac{a}{2}(1,1, \gamma)$. In order to use equation (4) we will compare the gradient terms along the $\mathrm{z}$ direction that is, we take $\hat{n}_{r}$ and $\hat{n}_{e}$ both equal to $\hat{z}$. We need to evaluate $\cos (\theta) \equiv \hat{R}_{\gamma} \cdot \hat{z}=\frac{\frac{\gamma}{2}}{\sqrt{\frac{1}{2}+\frac{\gamma^{2}}{4}}}$. For the neighbors $\vec{R}$, we use the previous expression with the sign changed (because they point opposite to the $\mathrm{z}$ direction) and with $\gamma=1$. Then the left hand side of equation (3) becomes (there are four contributions of each kind): 


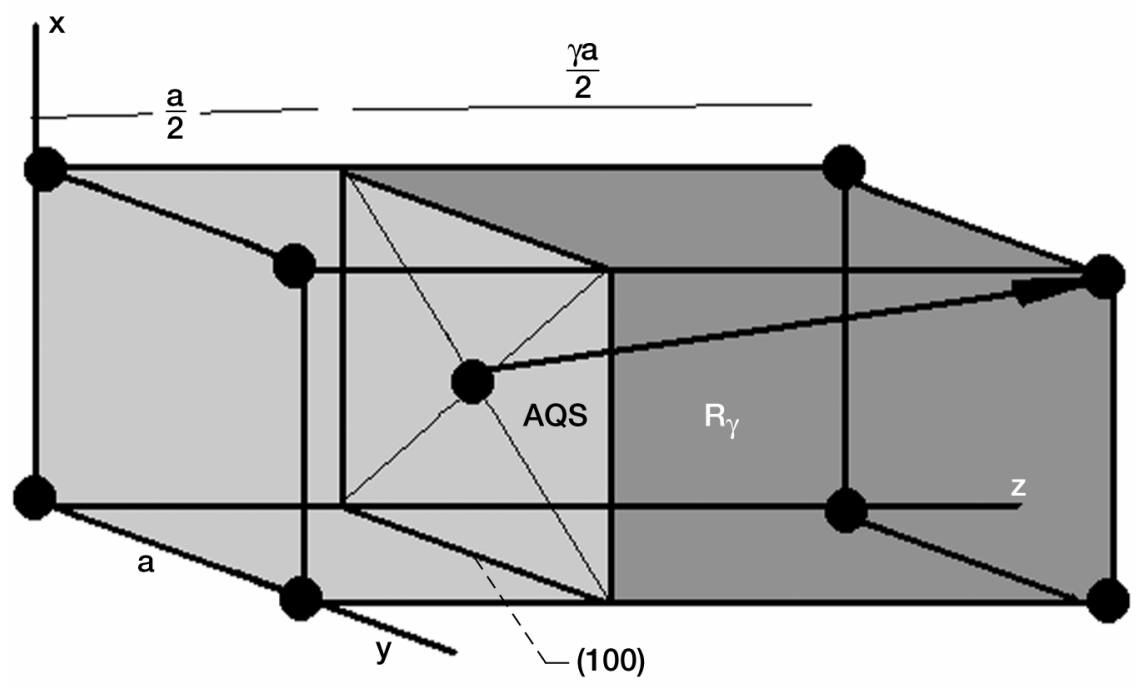

Figure 2.-The AQS in the quasi-free surface (100). The dark volume can only experience dilations controlled by $\mathrm{x}$. The light volume can experience both dilations and separations controlled by $\gamma$.

$$
\text { Equivalent Gradient }=-\frac{4}{\sqrt{3}}(\alpha R-p) R^{p-1} e^{-\alpha R}+\frac{2}{\sqrt{\frac{1}{2}+\frac{\gamma^{2}}{4}}}\left(\alpha R_{\gamma}-p\right) R_{\gamma}{ }^{p-1} e^{-\alpha R_{\gamma}}
$$

where $R=\frac{\sqrt{3}}{2} a_{0} x$, and $R_{\gamma}=a_{0} x \sqrt{\frac{1}{2}+\frac{\gamma^{2}}{4}}$.

The left hand side of equation (3) can be derived more simply in a similar way:

$$
\text { Equivalent Density }=4 R^{p} e^{-\alpha R}+4 R_{\gamma}^{p} e^{-\alpha R_{\gamma}}
$$

\section{Application of the Method}

We use the method described in the Generalization of UBER section to predict surface energy formations of $\mathrm{Fe}(110)$ and (111). It is necessary to select the reference surface which has the largest density gradient, that is the second term in equation (1). Fortunately, this information is available from reference [2]. With the advent in new ab initio techniques such information can be available for other surfaces of interest.

In order to solve equations (3) and (4) we need evaluate the density and gradient terms for the real crystals of interest and compare them with expressions given in equations (19) and (20).

1) $\mathrm{Fe}(110)$ surface energy formation.

Iron's ground state structure is BCC. Figure 3 shows a typical atom. When creating a surface (110) passing through that atom, two near neighbor atoms are lost. 


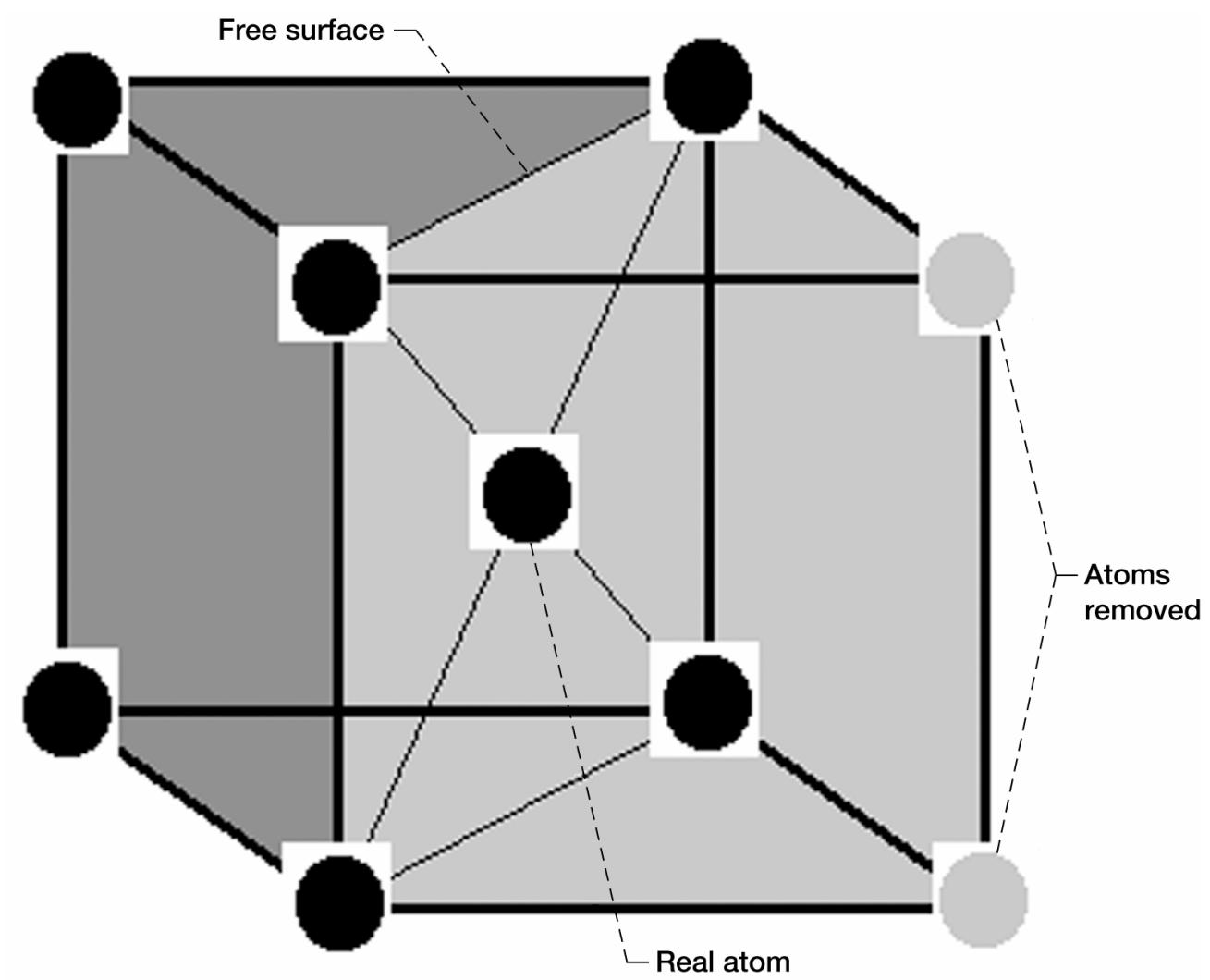

Figure 3.-An atom in the real crystal loses two near neighbors when creating a (110) BCC surface.

The contribution to the density from neighboring atoms is

$$
\operatorname{Density}(110)=6 R_{0}^{p} e^{-\alpha R_{0}}
$$

with $R_{0}=\frac{\sqrt{3}}{2} a_{0}$, and $\mathrm{a}_{0}$ the equilibrium lattice parameter.

To calculate the gradient term for BCC (110), we refer to figure 3. We notice that by symmetry the individual gradient terms from neighboring atoms in the (110) plane, when added together, cancel. Then the gradient term reduces to the contributions from the two remaining neighbors in the first layer below the free surface. By symmetry (they make the same angle with respect to the normal to the (110) plane) they both contribute to the sum on the right-hand side of equation (3) the same amount. One of those neighbors is at $\vec{R}=-\frac{a_{0}}{2}(1,1,1)$. The direction of the normal to (110) is $\hat{n}=\frac{(110)}{\sqrt{2}}$. Then $\frac{\vec{R}}{|\vec{R}|} \cdot \hat{n}=-\sqrt{\frac{2}{3}}$. Since the two atoms contribute the same, the gradient term becomes

$$
\operatorname{Gradient}(110)=-2 \sqrt{\frac{2}{3}}\left(\alpha R_{0}-p\right) R_{0}^{p-1} e^{-\alpha R_{0}}
$$


Next we use equations (19), (20), (21) and (22) and substitute in (3) and (4). From that we find the values of $\gamma$ and $x$ that satisfy the equations. Define $\Delta(x, \gamma)=\sqrt{[\text { Equivalent Density }- \text { Density }(110)]^{2}+[\text { Equivalent Gradient-Gradient }(110)]^{2}}$

Figure 4 shows a gray level plot of $\Delta(\mathrm{x}, \gamma)$ for the parameters of iron. From there a pair of roots is identified at $\mathrm{x}=0.959$ and $\gamma=1.119$. Substituting these values in equation (18) provides $\Delta \mathrm{E}_{\mathrm{AQS}}=2.49 \mathrm{~J} / \mathrm{m}^{2}$, which compares well with $2.247 \mathrm{~J} / \mathrm{m}^{2}$ from first principles [2, 3].

2) Fe (111) surface energy formation.

Figure 5 shows a typical atom. When creating a surface (111) passing through that atom, four near neighbor atoms are lost.

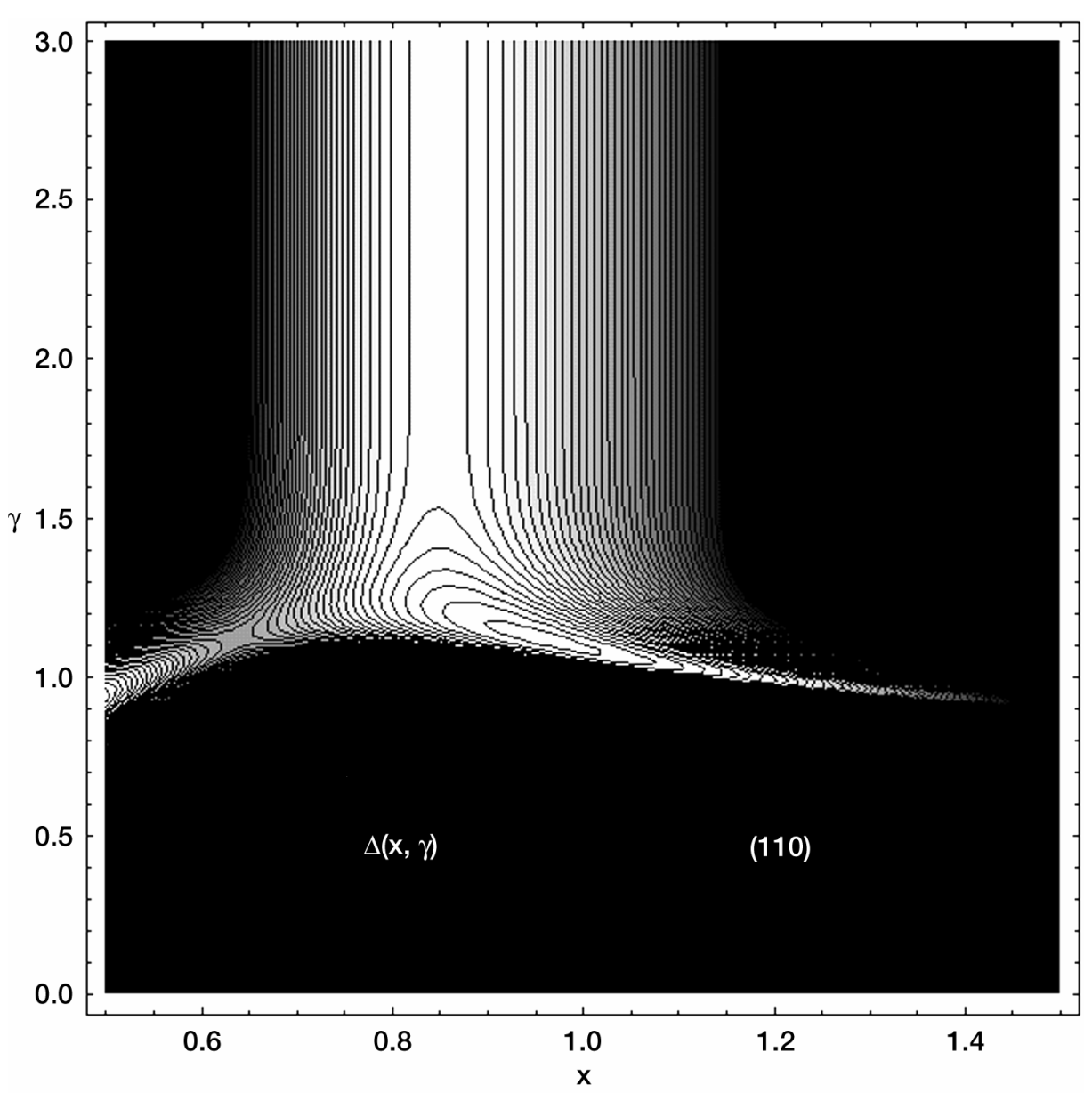

Figure 4.-Function to find the optimal values of $x$ and $\gamma$. 


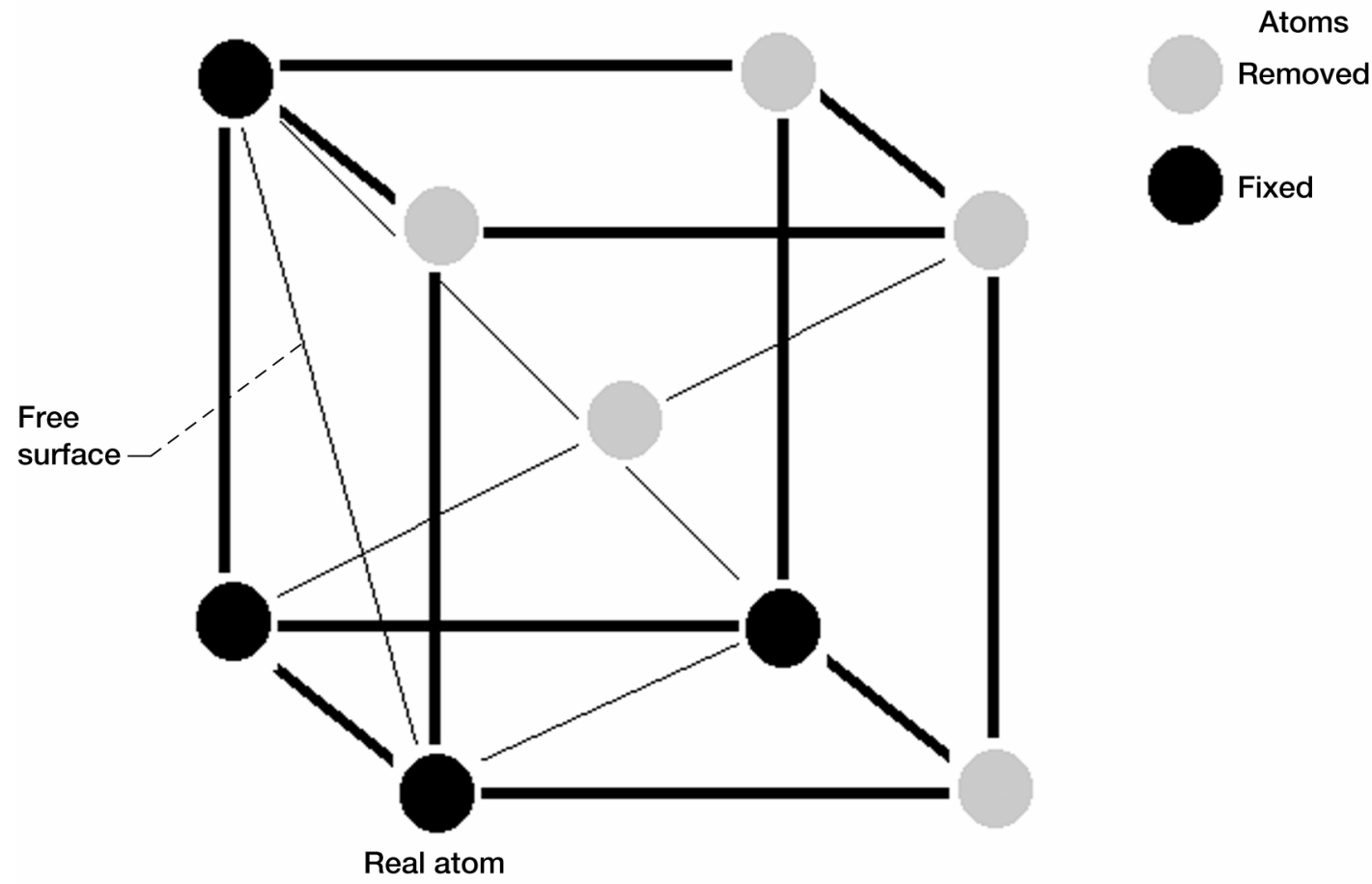

Figure 5.-An atom in the (111) Fe surface loses four near neighbors as compared with bulk.

The gradient term involves three atoms in the first layer below the surface with $\frac{\vec{R}}{|\vec{R}|} \cdot \hat{n}=-\frac{1}{3}$, and one atom in the second layer below the surface with $\frac{\vec{R}}{|\vec{R}|} \cdot \hat{n}=-1$. Then

$$
\operatorname{Gradient}(111)=-\left(3 \frac{1}{3}+1.1\right)\left(\alpha R_{0}-p\right) R_{0}^{p-1} e^{-\alpha R_{0}}=-2\left(\alpha R_{0}-p\right) R_{0}^{p-1} e^{-\alpha R_{0}}
$$

and

$$
\text { Density }(111)=4 R_{0}^{p} e^{-\alpha R_{0}}
$$

Next we use equations (19), (20), (23) and (24) and substitute in (3) and (4). From that we find the values of $\gamma$ and $x$ that satisfy the equations. Define as in 1) $\Delta(x, \gamma)=\sqrt{[\text { Equivalent Density-Density }(111)]^{2}+[\text { Equivalent Gradient-Gradient }(111)]^{2}}$

Figure 6 shows a gray level plot of $\Delta(\mathrm{x}, \gamma)$ for the parameters of iron. From there a pair of roots is identified at $\mathrm{x}=1.07006$ and $\gamma=1.19797$. Substituting these values in equation (18) provides $\Delta \mathrm{E}_{\mathrm{AQS}}=2.59 \mathrm{~J} / \mathrm{m}^{2}$, which also compares well with $2.62 \mathrm{~J} / \mathrm{m}^{2}$ from first principles [2, 3]. 


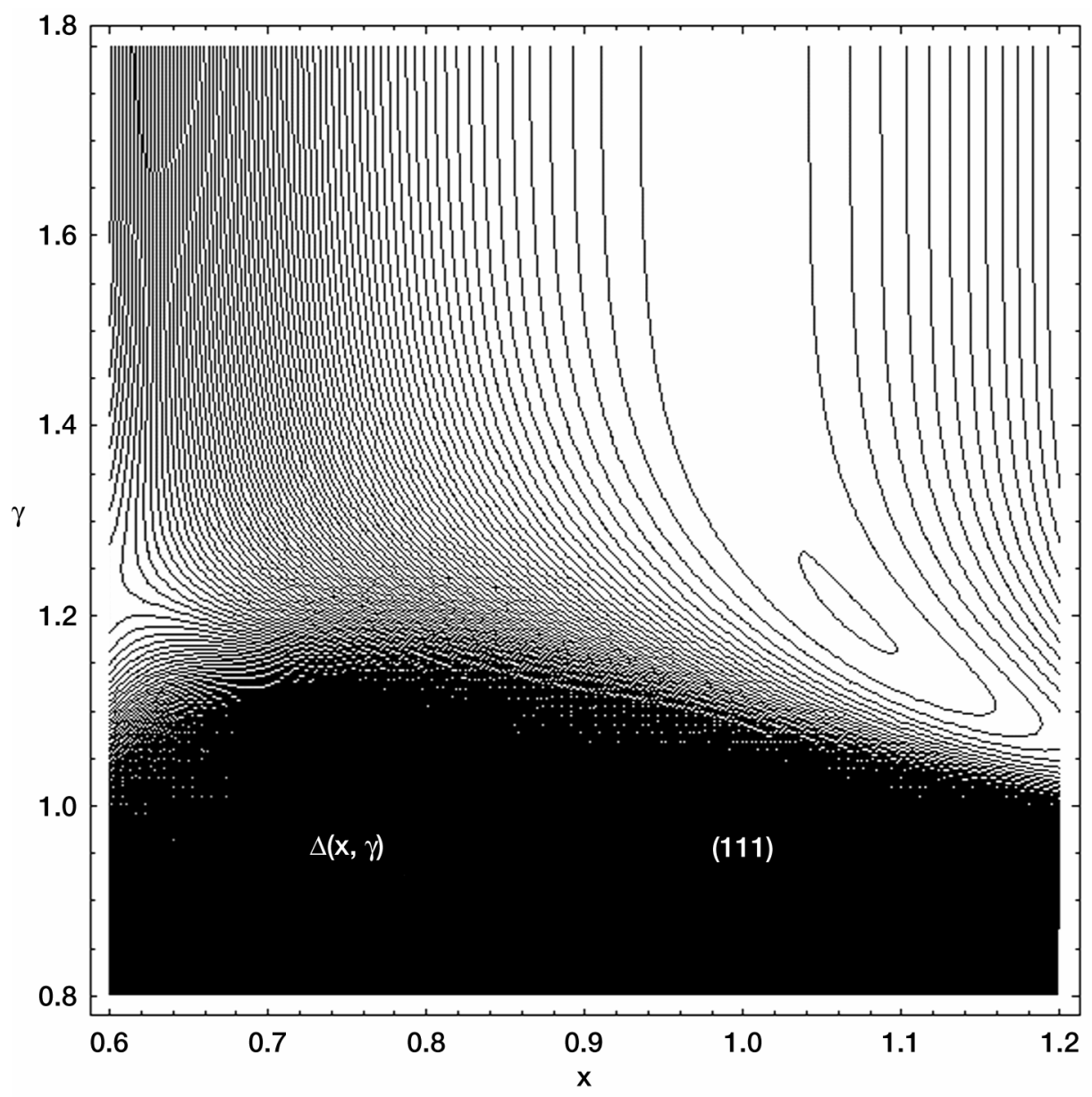

Figure 6.-Function to find the optimal values of $x$ and $\gamma$ for the formation of the (111) surface.

Table 1 shows results for Fe low index energy formation calculated by various methods. In addition, the table shows the ratios between (110) and (111) surface energies. This number is relevant because it has been used in the past as a gauge of the predictive power of a theory. It is known [8] that the ratios of surface energies are lower than expected from simple geometrical arguments based on surface roughness. This is due to the smoothing of the electron density parallel to the surface. Our results for the (111) to (110) energies are within $10 \%$ of the ab initio results.

Table I.-Comparison of surface energies calculated from various method.

\begin{tabular}{|c|c|c|c|}
\hline $\mathrm{J} / \mathrm{m}^{2}$ & OECT [5] & $\begin{array}{c}\text { AB INITIO } \\
{[2,3]}\end{array}$ & MEC (This work) \\
\hline$F e(110)$ & 1.78 & 2.25 & 2.49 \\
\hline$F e(111)$ & 2.48 & 2.62 & 2.59 \\
\hline$F e(110)$ & 1.39 & 1.16 & 1.04 \\
\hline$F e(111)$ & & & \\
\hline
\end{tabular}




\section{Conclusions}

This paper introduced MECT, whose main features are the introduction of a gradient term in the density and the possibility for anisotropic deformations of the equivalent lattice. This approach is of interest in the calculation of surface energies in particular, and more generally for low symmetry geometries in which the gradient of the density is significant and therefore makes an important contribution to the total energy. We have applied the method to calculate low index surface energy formation of $\mathrm{Fe}$ and found an improved agreement with ab initio results.

\section{References}

[1] W. Kohn, L. J. Sham, "Self-Consistent Equations Including Exchange and Correlation Effects", Phys. Rev. 140, A1133-A1138 (1965)

[2] M.J.S. Spencer, A. Hung, I.K. Snook, I. Yarovsky, "Density functional theory study of the relaxation and energy of iron surfaces", to appear in Surface Science (2002)

[3] A. Hung, I. Yarovsky, J. Muscat, S. Russo, I. Snook, R.O. Watts, "First-principles study of metallic iron interfaces", to appear in Surface Science.

[4] J. Ferrante, J.R. Smith, "A theory of adhesion at a bimetallic interface: overlap effects", Surface Science 38 (1973) 77-92

[5] A.M. Rodríguez, G. Bozzolo, J. Ferrante, "Multilayer relaxation and surface energies of fcc and bcc metals using equivalent crystal theory", Surface Science 289 (1993) 100-126

[6] J. R. Smith, A. Banerjea, T. Perry, J. Ferrante, G. Bozzolo, "Equivalent Crystal Theory of Metals and Semiconductors Defects", Phys. Rev. B44 (1991) 6444

[7] A. Banerjea and J.R. Smith, "Origins of the universal binding-energy relation", Phys. Rev. B 37, 6632-6645 (1988)

[8] M. Methfessel, D. Hennig, and M. Scheffler, "Trends of the surface relaxation, surface energies, and work functions of the 4d transition metal", Phys. Rev. B 46, 4816-4829 (1992) 


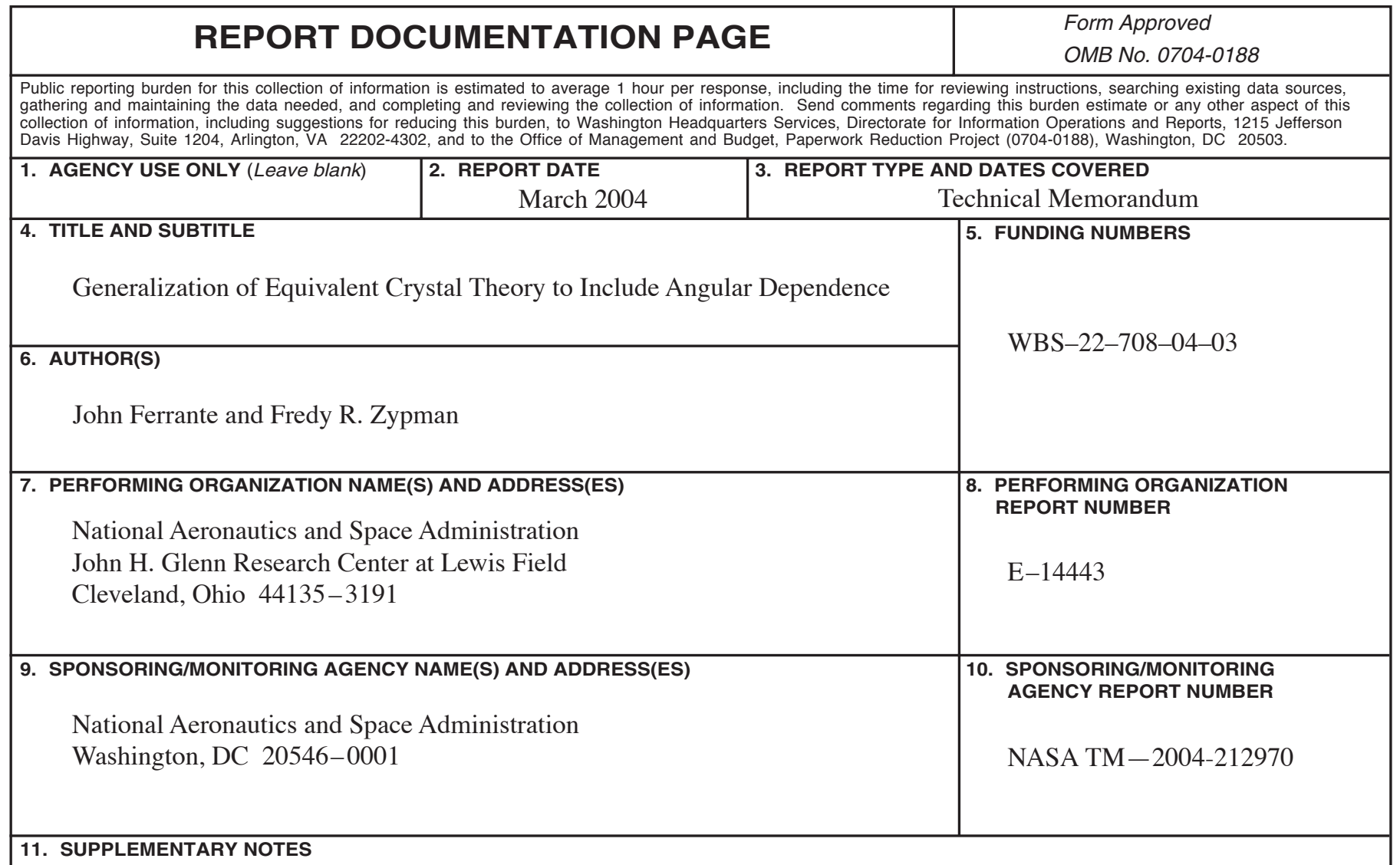

John Ferrante, NASA Glenn Research Center (retired); and Fredy R. Zypman, Yeshiva University, Department of Physics, 2495 Amsterdam Avenue, New York, New York 10033. Responsible person, Phillip B. Abel, organization code 5960, 216-433-6063.

12a. DISTRIBUTION/AVAILABILITY STATEMENT 12b. DISTRIBUTION CODE

Unclassified - Unlimited

Subject Categories: 72, 76, and 77

Distribution: Nonstandard

Available electronically at http://gltrs.grc.nasa.gov

This publication is available from the NASA Center for AeroSpace Information, 301-621-0390.

13. ABSTRACT (Maximum 200 words)

In the original Equivalent Crystal Theory, each atomic site in the real crystal is assigned an equivalent lattice constant, in general different from the ground state one. This parameter corresponds to a local compression or expansion of the lattice. The basic method considers these volumetric transformations and, in addition, introduces the possibility that the reference lattice is anisotropically distorted. These distortions however, were introduced ad-hoc. In this work, we generalize the original Equivalent Crystal Theory by systematically introducing site-dependent directional distortions of the lattice, whose corresponding distortions account for the dependence of the energy on anisotropic local density variations. This is done in the spirit of the original framework, but including a gradient term in the density. This approach is introduced to correct a deficiency in the original Equivalent Crystal Theory and other semiempirical methods in quantitatively obtaining the correct ratios of the surface energies of low index planes of cubic metals - (100), (110), and (111). We develop here the basic framework, and apply it to the calculation of Fe (110) and Fe (111) surface energy formation. The results, compared with first principles calculations, show an improvement over previous semiempirical approaches.

\begin{tabular}{|c|c|c|}
\hline \multicolumn{3}{|c|}{$\begin{array}{l}\text { 14. SUBJECT TERMS } \\
\text { Crystal structure; Metals; Iron; Surface }\end{array}$} \\
\hline $\begin{array}{l}\text { 17. SECURITY CLASSIFICATION } \\
\text { OF REPORT } \\
\text { Unclassified }\end{array}$ & $\begin{array}{l}\text { 18. SECURITY CLASSIFICATION } \\
\text { OF THIS PAGE } \\
\text { Unclassified }\end{array}$ & $\begin{array}{l}\text { 19. SECURITY CLASSIFICATION } \\
\text { OF ABSTRACT } \\
\text { Unclassified }\end{array}$ \\
\hline
\end{tabular}

NSN 7540-01-280-5500

Standard Form 298 (Rev. 2-89)

Prescribed by ANSI Std. Z39-18
$298-102$

15. NUMBER OF PAGES

18

20. LIMITATION OF ABSTRACT 